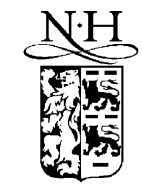

\title{
Exact complexity of Exact-Four-Colorability ${ }^{\text {* }}$
}

\author{
Jörg Rothe \\ Abteilung für Informatik, Heinrich-Heine-Universität Düsseldorf, 40225 Düsseldorf, Germany \\ Received 16 May 2002; received in revised form 26 January 2003 \\ Communicated by A.A. Bertossi
}

\begin{abstract}
Let $M_{k}$ be a given set of $k$ integers. Define Exact- $M_{k}$-Colorability to be the problem of determining whether or not $\chi(G)$, the chromatic number of a given graph $G$, equals one of the $k$ elements of the set $M_{k}$ exactly. In 1987, Wagner [Theoret. Comput. Sci. 51 (1987) 53-80] proved that Exact- $M_{k}$-Colorability is $\mathrm{BH}_{2 k}(\mathrm{NP})$-complete, where $M_{k}=\{6 k+1,6 k+3, \ldots, 8 k-1\}$ and $\mathrm{BH}_{2 k}(\mathrm{NP})$ is the $2 k$ th level of the Boolean hierarchy over NP. In particular, for $k=1$, it is DP-complete to determine whether or not $\chi(G)=7$, where $\mathrm{DP}=\mathrm{BH}_{2}(\mathrm{NP})$. Wagner raised the question of how small the numbers in a $k$-element set $M_{k}$ can be chosen such that Exact- $M_{k}$-Colorability still is $\mathrm{BH}_{2 k}(\mathrm{NP})$-complete. In particular, for $k=1$, he asked if it is DP-complete to determine whether or not $\chi(G)=4$.

In this note, we solve Wagner's question and prove the optimal result: For each $k \geqslant 1$, Exact $-M_{k}$-Colorability is $\mathrm{BH}_{2 k}(\mathrm{NP})$-complete for $M_{k}=\{3 k+1,3 k+3, \ldots, 5 k-1\}$. In particular, for $k=1$, we determine the precise threshold of the parameter $t \in\{4,5,6,7\}$ for which the problem Exact-\{t\}-Colorability jumps from NP to DP-completeness: It is DP-complete to determine whether or not $\chi(G)=4$, yet Exact-\{3\}-Colorability is in NP.
\end{abstract}

() 2003 Elsevier Science B.V. All rights reserved.

Keywords: Computational complexity; Graph colorability; Completeness; Boolean hierarchy

\section{Exact colorability and the Boolean hierarchy over NP}

To classify the complexity of problems known to be NP-hard or coNP-hard, but seemingly not contained in NP $\cup$ coNP, Papadimitripu and Yannakakis [16] in-

This work was supported in part by grant NSF-INT9815095/DAAD-315-PPP-gü-ab and by a Heisenberg Fellowship of the Deutsche Forschungsgemeinschaft. The results of this paper appear in preliminary form in the Proceedings of the 2nd IFIP International Conference on Theoretical Computer Science (TCS 2002), which was held in conjunction with the 17th IFIP World Computer Congress in Montréal, Québec, Canada.

E-mail address: rothe@cs.uni-duesseldorf.de (J. Rothe). troduced DP, the class of differences of two NP problems. They showed that DP contains various interesting types of problems, including uniqueness problems, critical graph problems, and exact optimization problems. For example, Cai and Meyer [7] proved the DPcompleteness of Minimal-3-Uncolorability, a critical graph problem that asks whether a given graph is not 3-colorable, but deleting any of its vertices makes it 3-colorable. A graph is said to be $k$-colorable if its vertices can be colored using no more than $k$ colors such that no two adjacent vertices receive the same color. The chromatic number of a graph $G$, denoted $\chi(G)$, is defined to be the smallest $k$ such that $G$ is $k$-colorable. 
Generalizing DP, Cai et al. [3,4] defined and studied the Boolean hierarchy over NP. Their papers initiated an intensive work and many papers on the Boolean hierarchy; e.g., $[20,15,13,21,2,5,1,6,12,17]$ to name just a few.

To define the Boolean hierarchy, we use the symbols $\wedge$ and $\vee$, respectively, to denote the complex intersection and the complex union of set classes. That is, for classes $\mathcal{C}$ and $\mathcal{D}$ of sets, define

$\mathcal{C} \wedge \mathcal{D}=\{A \cap B \mid A \in \mathcal{C}$ and $B \in \mathcal{D}\} ;$

$\mathcal{C} \vee \mathcal{D}=\{A \cup B \mid A \in \mathcal{C}$ and $B \in \mathcal{D}\}$.

Definition 1 (Cai et al. [3]). The Boolean hierarchy over NP is inductively defined as follows:

$\mathrm{BH}_{1}(\mathrm{NP})=\mathrm{NP}$,

$\mathrm{BH}_{2}(\mathrm{NP})=\mathrm{NP} \wedge \mathrm{coNP}$,

$\mathrm{BH}_{k}(\mathrm{NP})=\mathrm{BH}_{k-2}(\mathrm{NP}) \vee \mathrm{BH}_{2}(\mathrm{NP})$ for $k \geqslant 3$,

and

$\mathrm{BH}(\mathrm{NP})=\bigcup_{k \geqslant 1} \mathrm{BH}_{k}(\mathrm{NP})$.

Equivalent definitions in terms of different Boolean hierarchy normal forms can be found in the papers [3, 20,15]; for the Boolean hierarchy over arbitrary set rings, we refer to the early work by Hausdorff [11]. Note that $\mathrm{DP}=\mathrm{BH}_{2}(\mathrm{NP})$.

All hardness and completeness results in this paper are with respect to the polynomial-time many-one reducibility, denoted by $\leqslant_{\mathrm{m}}^{\mathrm{p}}$, which is defined as follows. For sets $A$ and $B, A \leqslant \mathrm{~m} B$ if and only if there exists a polynomial-time computable function $f$ such that for each $x \in \Sigma^{*}, x \in A$ if and only if $f(x) \in B$. A set $B$ is said to be $\mathcal{C}$-hard for a complexity class $\mathcal{C}$ if and only if $A \leqslant \mathrm{~m} \quad B$ for each $A \in \mathcal{C}$. A set $B$ is said to be $\mathcal{C}$-complete if and only if $B$ is $\mathcal{C}$-hard and $B \in \mathcal{C}$.

In his seminal paper [20], Wagner provided sufficient conditions to prove problems complete for the levels of the Boolean hierarchy. In particular, he established the following lemma for $\mathrm{BH}_{2 k}(\mathrm{NP})$.

Lemma 2 (Wagner, see Theorem 5.1(3) of [20]). Let A be some NP-complete problem, let B be an arbitrary problem, and let $k \geqslant 1$ be fixed.
If there exists a polynomial-time computable function $f$ such that, for all strings $x_{1}, x_{2}, \ldots, x_{2 k} \in \Sigma^{*}$ satisfying $(\forall j: 1 \leqslant j<2 k)\left[x_{j+1} \in A \Rightarrow x_{j} \in A\right]$, it holds that

$\left\|\left\{i \mid x_{i} \in A\right\}\right\|$ is odd $\Leftrightarrow f\left(x_{1}, x_{2}, \ldots, x_{2 k}\right) \in B$,

then $B$ is $\mathrm{BH}_{2 k}(\mathrm{NP})$-hard.

For fixed $k \geqslant 1$, let $M_{k}=\{6 k+1,6 k+3, \ldots$, $8 k-1\}$, and define the problem

$$
\begin{aligned}
& \text { Exact }-M_{k} \text {-Colorability } \\
& \quad=\left\{G \mid G \text { is a graph with } \chi(G) \in M_{k}\right\} .
\end{aligned}
$$

In particular, Wagner applied Lemma 2 to prove that, for each $k \geqslant 1$, Exact-M $M_{k}$-Colorability is $\mathrm{BH}_{2 k}(\mathrm{NP})$-complete. For the special case of $k=1$, it follows that Exact- $\{7\}$-Colorability is DPcomplete.

Wagner [20, p. 70] raised the question of how small the numbers in a $k$-element set $M_{k}$ can be chosen such that Exact $-M_{k}$-Colorability still is $\mathrm{BH}_{2 k}(\mathrm{NP})$-complete. Consider the special case of $k=$ 1. It is easy to see that Exact-\{3\}-Colorability is in NP and, thus, cannot be DP-complete unless the Boolean hierarchy collapses; see Proposition 3 below. Consequently, for $k=1$, Wagner's result leaves a gap in determining the precise threshold $t \in\{4,5,6,7\}$ for which the problem Exact-\{t\}-Colorability jumps from NP- to DP-completeness.

Closing this gap and solving Wagner's question, we show that for each $k \geqslant 1$, Exact- $M_{k}$-Colorability is $\mathrm{BH}_{2 k}(\mathrm{NP})$-complete for $M_{k}=\{3 k+1$, $3 k+3, \ldots, 5 k-1\}$. In particular, for $k=1$, it is DPcomplete to determine whether or not $\chi(G)=4$.

\section{Solving Wagner's question}

Proposition 3. Fix any $k \geqslant 1$, and let $M_{k}$ be any set that contains $k$ noncontiguous positive integers including 3. Then, Exact $-M_{k}$-Colorability is in $\mathrm{BH}_{2 k-1}(\mathrm{NP})$; in particular, for $k=1$, Exact $-\{3\}-$ Colorability is in NP.

Hence, Exact-M $M_{k}$-Colorability is not $\mathrm{BH}_{2 k}(\mathrm{NP})$-complete unless the Boolean hierarchy, and consequently the polynomial hierarchy, collapses. 
Proof. Fix any $k \geqslant 1$, and let $M_{k}$ be given as above. Note that

$$
\begin{aligned}
& \text { Exact }-M_{k} \text {-Colorability } \\
& =\bigcup_{i \in M_{k}} \text { Exact- }\{i\} \text {-Colorability. }
\end{aligned}
$$

Since for each $i \in M_{k}$,

$$
\begin{aligned}
& \text { Exact- }\{i\} \text {-Colorability } \\
& \quad=\{G \mid \chi(G) \leqslant i\} \cap\{G \mid \chi(G)>i-1\}
\end{aligned}
$$

and since the set $\{G \mid \chi(G) \leqslant i\}$ is in NP and the set $\{G \mid \chi(G)>i-1\}$ is in coNP, each of the $k-1$ sets Exact-\{i\}-Colorability with $i \in M_{k}-\{3\}$ is in DP. However, Exact-\{3\}-Colorability is even contained in NP, since it can be checked in polynomial time whether a given graph is 2-colorable, so $\{G \mid \chi(G)>2\}$ is in P. Hence, Exact $-M_{k}-\mathrm{Col}-$ orability is in $\mathrm{BH}_{2 k-1}(\mathrm{NP})$.

To prove the main result of this paper, we apply two known reductions from 3-SAT to 3-Colorability, which have certain useful properties needed to apply Lemma 2 . These properties are stated in the following two lemmas.

The first reduction is the standard reduction from 3-SAT to 3-Colorability, which is due to Garey, Johnson, and Stockmeyer $[9,19]$. Here, $3-\mathrm{SAT}$ is the satisfiability problem for Boolean formulas in conjunctive normal form and with three literals per clause, and 3-Colorability is the set of graphs $G$ with $\chi(G) \leqslant 3$. Both are standard NP-complete problems [8].

Lemma 4 (Garey et al. $[9,19])$. There exists a polynomial-time computable function $\sigma$ that $\leqslant_{\mathrm{m}}^{\mathrm{p}}$-reduces 3SAT to 3-Colorability and satisfies the following two properties:

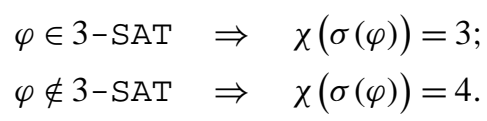

The second reduction is due to Guruswami and Khanna [10]. Using the PCP theorem, Khanna, Linial, and Safra [14] showed that it is NP-hard to color a 3 -colorable graph with only four colors. Guruswami and Khanna [10] gave a novel proof of the same result that does not rely on the PCP theorem. Theorem 6 below uses the properties of their direct transformation, which are stated in Lemma 5.

Lemma 5 (cf. the proof of Theorem 1 of [10]). There exists a polynomial-time computable function $\rho$ that $\leqslant_{\mathrm{m}}^{\mathrm{p}}$-reduces 3-SAT to 3-Colorability and satisfies the following two properties:

$$
\begin{aligned}
& \varphi \in 3-\mathrm{SAT} \Rightarrow \chi(\rho(\varphi))=3 \text {; } \\
& \varphi \notin 3-\mathrm{SAT} \Rightarrow \chi(\rho(\varphi))=5 \text {. }
\end{aligned}
$$

Proof. The Guruswami-Khanna reduction, call it $\rho$, is the composition of two subsequent reductions: first a reduction from 3-SAT to the independent set problem, another standard NP-complete problem [8]; and then from the independent set problem to 3Colorability. The independent set problem asks, given graph a $G$ and an integer $m$, whether or not the size of a maximum independent set of $G$ (i.e., of a maximum subset of $G$ 's vertex set in which no two vertices are adjacent) is at least $m$.

We omit presenting the details of Guruswami and Khanna's very sophisticated construction, which involves tree-like structures and various types of gadgets connecting them. Instead, we give only a rough outline of the construction. Using the standard reduction from 3-SAT to the independent set problem [8], construct from the given Boolean formula $\varphi$ a graph $G$ consisting of $m$ triangles (i.e., of $m$ cliques of size 3 each) such that each triangle corresponds to some clause of $\varphi$ and the vertices of any two distinct triangles are connected by an edge if and only if they represent some literal and its negation, respectively, in the corresponding clauses.

Then, transform $G$ to a graph $H=\rho(\varphi)$ such that, to each such triangle in $G$, there corresponds a tree-like structure with three leaves in the graph $H$. The "vertices" of the tree-like structures are basic templates consisting of $3 \times 3$ grids such that the vertices in each row and in each column of the grid induce a 3-clique. The three vertices in the first column of any such basic template are shared among all the basic templates in each of the tree-like structures. Finally, connect the leaves of any two distinct tree-like structures by appropriate gates that will be described later on. 
Similarly, we also omit presenting the details of their clever proof of correctness and give only a rough outline of the idea. Intuitively, it is argued that every 4-coloring of the graph $H$ "selects" the root of each tree-like structure and that this root selection is inherited downwards to the leaves. Then, the gadgets connecting the tree-like structures at the leaf-level ensure that if the graph $H=\rho(\varphi)$ is 4-colorable, then $\varphi$ is satisfiable. On the other hand, it is proven that if $\varphi$ is satisfiable, then $H$ is even 3-colorable. Thus, the construction guarantees that an unsatisfiable formula implies a graph with chromatic number at least five. In other words, the graph $H$ has never a chromatic number of exactly four, no matter whether or not $\varphi$ is satisfiable.

However, there is one subtle point in the Guruswami-Khanna reduction that requires detailed explanation here, since it is crucial to our application of their reduction in Theorem 6. As noted above, Guruswami and Khanna [10] prove that their reduction $\rho$ satisfies that:

- $\varphi \in$ 3-SAT implies $\chi(\rho(\varphi))=3$, which is Eq. (2.3), and

- $\varphi \notin 3-$ SAT implies $\chi(\rho(\varphi)) \geqslant 5$.

Guruswami and Khanna [10] note that the graph $H=\rho(\varphi)$ they construct is always 6-colorable. However, to apply Wagner's technique (see Lemma 2) in the proof of Theorem 6 , we need to have that $\varphi \notin$ $3-$ SAT implies not only $5 \leqslant \chi(H) \leqslant 6$, but exactly $\chi(H)=5$.

We now argue that the Guruswami-Khanna construction even gives that the graph $H$ is always 5colorable as required. To see why, look at the reduction in [10]. Recall that the graph $H$ consists of tree-like structures whose vertices are replaced by basic templates, which are $3 \times 3$ grids whose rows and columns induce 3 -cliques. Thus, the basic templates can always be colored with three colors, say 1,2 , and 3 . In addition, some leaves of the tree-like structures are connected by leaf-level gadgets of two types, the "same row kind" and the "different row kind".

The latter gadgets consist of two vertices connected to some grids, and thus can always be colored with two additional colors. The leaf-level gadgets of the "same row kind" consist of a triangle whose vertices are adjacent to two grid vertices each. Hence, regardless of which 3-coloring is used for the grids, one can always color one triangle vertex, say $t_{1}$, with a color $c \in\{1,2,3\}$ such that $c$ is different from the colors of the two grid vertices adjacent to $t_{1}$. Using two additional colors for the other two triangle vertices implies $\chi(H) \leqslant 5$, which proves Eq. (2.4).

Theorem 6. For each fixed $k \geqslant 1$, let $M_{k}=\{3 k+1$, $3 k+3, \ldots, 5 k-1\}$. Then, Exact-M $M_{k}$-Colorability is $\mathrm{BH}_{2 k}(\mathrm{NP})$-complete.

Proof. Apply Lemma 2 with $A$ being the NP-complete problem 3-SAT and $B$ being the problem Exact$M_{k}$-Colorability, where $M_{k}=\{3 k+1,3 k+3$, $\ldots, 5 k-1\}$ for fixed $k$.

Let $\sigma$ be the standard reduction from 3-SAT to 3Colorability according to Lemma 4 , and let $\rho$ be the Guruswami-Khanna reduction from 3-SAT to 3Colorability according to Lemma 5.

The join operation $\oplus$ on graphs is defined as follows: Given two disjoint graphs $A=\left(V_{A}, E_{A}\right)$ and $B=\left(V_{B}, E_{B}\right)$, their join $A \oplus B$ is the graph with vertex set $V_{A \oplus B}=V_{A} \cup V_{B}$ and edge set $E_{A \oplus B}=$ $E_{A} \cup E_{B} \cup\left\{\{a, b\} \mid a \in V_{A}\right.$ and $\left.b \in V_{B}\right\}$. Note that $\oplus$ is an associative operation on graphs and $\chi(A \oplus B)=$ $\chi(A)+\chi(B)$.

Let $\varphi_{1}, \varphi_{2}, \ldots, \varphi_{2 k}$ be $2 k$ given Boolean formulas satisfying $\varphi_{j+1} \in 3-\mathrm{SAT} \Rightarrow \varphi_{j} \in 3-\mathrm{SAT}$ for each $j$ with $1 \leqslant j<2 k$. Define $2 k$ graphs $H_{1}, H_{2}, \ldots, H_{2 k}$ as follows. For each $i$ with $1 \leqslant i \leqslant k$, define $H_{2 i-1}=$ $\rho\left(\varphi_{2 i-1}\right)$ and $H_{2 i}=\sigma\left(\varphi_{2 i}\right)$. By Eqs. (2.1)-(2.4), it follows that:

$$
\chi\left(H_{j}\right)=\left\{\begin{array}{cc}
3 \quad & \text { if } 1 \leqslant j \leqslant 2 k \text { and } \varphi_{j} \in 3-\mathrm{SAT}, \\
4 \quad & \text { if } j=2 i \text { for some } i \in\{1,2, \ldots, k\} \\
& \text { and } \varphi_{j} \notin 3-\mathrm{SAT}, \\
5 \quad \begin{array}{c}
\text { if } j=2 i-1 \text { for some } i \in\{1,2, \ldots, k\} \\
\text { and } \varphi_{j} \notin 3-\mathrm{SAT} .
\end{array}
\end{array}\right.
$$

For each $i$ with $1 \leqslant i \leqslant k$, define the graph $G_{i}$ to be the disjoint union of the graphs $H_{2 i-1}$ and $H_{2 i}$. Thus, $\chi\left(G_{i}\right)=\max \left\{\chi\left(H_{2 i-1}\right), \chi\left(H_{2 i}\right)\right\}$, for each $i$ with $1 \leqslant i \leqslant k$. The construction of our reduction $f$ is completed by defining $f\left(\varphi_{1}, \varphi_{2}, \ldots, \varphi_{2 k}\right)=G$, where the graph $G=\bigoplus_{i=1}^{k} G_{i}$ is the join of the graphs $G_{1}, G_{2}, \ldots, G_{k}$. Thus, 
$\chi(G)=\sum_{i=1}^{k} \chi\left(G_{i}\right)=\sum_{i=1}^{k} \max \left\{\chi\left(H_{2 i-1}\right), \chi\left(H_{2 i}\right)\right\}$.

It follows from our construction that

$\left\|\left\{i \mid \varphi_{i} \in 3-\mathrm{SAT}\right\}\right\|$ is odd

$$
\begin{aligned}
& \Longleftrightarrow(\exists i: 1 \leqslant i \leqslant k) \\
& {\left[\varphi_{1}, \ldots, \varphi_{2 i-1} \in 3-\mathrm{SAT}\right. \text { and }} \\
& \left.\varphi_{2 i}, \ldots, \varphi_{2 k} \notin 3-\mathrm{SAT}\right] \\
& \stackrel{(2.6),(2.7)}{\Longleftrightarrow}(\exists i: 1 \leqslant i \leqslant k) \\
& {\left[\sum_{j=1}^{k} \chi\left(G_{j}\right)=3(i-1)+4+5(k-i)\right.} \\
& =5 k-2 i+1] \\
& \stackrel{(2.7)}{\Longleftrightarrow} \chi(G) \in M_{k}=\{3 k+1,3 k+3, \ldots, 5 k-1\} \\
& \Longleftrightarrow f\left(\varphi_{1}, \varphi_{2}, \ldots, \varphi_{2 k}\right) \\
& =G \in \text { Exact }-M_{k} \text {-Colorability. }
\end{aligned}
$$

Hence, Eq. (1.1) is satisfied. By Lemma 2, Exact$M_{k}$-Colorability is $\mathrm{BH}_{2 k}(\mathrm{NP})$-complete.

In particular, for $k=1$, Theorem 6 has the following corollary.

Corollary 7. Exact-\{4\}-Colorability is DPcomplete.

To conclude this paper, we mention in passing that Riege and this author [17] recently obtained similar $\mathrm{BH}_{2 k}(\mathrm{NP})$-completeness results for the exact versions of the domatic number problem and the conveyor flow shop problem.

The results of this paper appear in preliminary form in [18].

\section{Acknowledgements}

Interesting discussions with Klaus Wagner, Venkatesan Guruswami, Edith and Lane Hemaspaandra, Harald Hempel, Dieter Kratsch, Tobias Riege, and Gerd Wechsung are gratefully acknowledged. I also thank the anonymous IFIP-TCS 2002 and IPL referees for their nice comments, and I thank Alan Bertossi for his guidance through the editorial process.

\section{References}

[1] R. Beigel, R. Chang, M. Ogiwara, A relationship between difference hierarchies and relativized polynomial hierarchies, Math. Systems Theory 26 (3) (1993) 293-310.

[2] R. Beigel, Bounded queries to SAT and the Boolean hierarchy, Theoret. Comput. Sci. 84 (2) (1991) 199-223.

[3] J. Cai, T. Gundermann, J. Hartmanis, L. Hemachandra, V. Sewelson, K. Wagner, G. Wechsung, The Boolean hierarchy I: Structural properties, SIAM J. Comput. 17 (6) (1988) 12321252.

[4] J. Cai, T. Gundermann, J. Hartmanis, L. Hemachandra, V. Sewelson, K. Wagner, G. Wechsung, The Boolean hierarchy II: Applications, SIAM J. Comput. 18 (1) (1989) 95-111.

[5] R. Chang, On the structure of NP computations under Boolean operators, PhD thesis, Cornell University, Ithaca, NY, 1991.

[6] R. Chang, J. Kadin, The Boolean hierarchy and the polynomial hierarchy: A closer connection, SIAM J. Comput. 25 (2) (1996) 340-354.

[7] J. Cai, G. Meyer, Graph minimal uncolorability is $\mathrm{D}^{\mathrm{P}}$-complete, SIAM J. Comput. 16 (2) (1987) 259-277.

[8] M. Garey, D. Johnson, Computers and Intractability: A Guide to the Theory of NP-Completeness, W.H. Freeman and Company, New York, 1979.

[9] M. Garey, D. Johnson, L. Stockmeyer, Some simplified NPcomplete graph problems, Theoret. Comput. Sci. 1 (1976) 237-267.

[10] V. Guruswami, S. Khanna, On the hardness of 4-coloring a 3-colorable graph, in: Proceedings of the 15th Annual IEEE Conference on Computational Complexity, IEEE Computer Society Press, May 2000, pp. 188-197.

[11] F. Hausdorff, Grundzüge der Mengenlehre, Walter de Gruyter, Berlin, 1914.

[12] L. Hemaspaandra, J. Rothe, Unambiguous computation: Boolean hierarchies and sparse Turing-complete sets, SIAM J. Comput. 26 (3) (1997) 634-653.

[13] J. Kadin, The polynomial time hierarchy collapses if the Boolean hierarchy collapses, SIAM J. Comput. 17 (6) (1988) 1263-1282; Erratum, SIAM J. Comput. 20 (2) (1991) 404.

[14] S. Khanna, N. Linial, S. Safra, On the hardness of approximating the chromatic number, Combinatorica 20 (3) (2000) 393415.

[15] J. Köbler, U. Schoning, K. Wagner, The difference and truthtable hierarchies for NP, RAIRO Inform. Théor. Appl. 21 (1987) 419-435.

[16] C. Papadimitriou, M. Yannakakis, The complexity of facets (and some facets of complexity), J. Comput. System Sci. 28 (2) (1984) 244-259.

[17] T. Riege, J. Rothe, Complexity of the exact domatic number problem and of the exact conveyor flow shop problem, Technical Report cs.CC/0212016, Computing Research Repository (CoRR), December 2002, 14 pp.; Available on-line at http://xxx.lanl.gov/abs/cs.CC/0212016.

[18] J. Rothe, H. Spakowski, J. Vogel, Exact complexity of ExactFour-Colorability and of the winner problem for Young elections, in: R. Baeza-Yates, U. Montanari, N. Santoro (Eds.), Foundations of Information Technology in the Era of Network 
and Mobile Computing, Proceedings of the 2nd IFIP International Conference on Theoretical Computer Science, Stream 1 of the 17th IFIP World Computer Congress, Kluwer Academic Publishers, August 2002, pp. 310-322.

[19] L. Stockmeyer, Planar 3-colorability is NP-complete, SIGACT News 5 (3) (1973) 19-25.
[20] K. Wagner, More complicated questions about maxima and minima, and some closures of NP, Theoret. Comput. Sci. 51 (1987) 53-80.

[21] K. Wagner, Bounded query classes, SIAM J. Comput. 19 (5) (1990) 833-846. 